\title{
Does co-treatment with immunosuppressors improve outcome in patients with Crohn's disease treated with adalimumab?
}

\author{
C. Reenaers ${ }^{\star}$, E. Louis ${ }^{\star}$, J. Belaiche*, L. Seidel ${ }^{\dagger}$, S. Keshav ${ }^{\star} \&$ S. Travis ${ }^{\star}$
}

${ }^{\star}$ Gastroenterology Department, $\mathrm{CHU}$ Sart Tilman, Liège University, Liège, Belgium.

†Medical Informatics and Biostatistic Department, CHU Sart Tilman, Liège University, Liège, Belgium.

*Translational Gastroenterology Unit, John Radcliffe Hospital, Oxford, UK.

\section{Correspondence to:}

Dr C. Reenaers, Hepato-

gastroenterology Department, CHU

Sart-Tilman, 4000 Liège, Belgium.

E-mail: catherinereenaers@hotmail.com

\section{Publication data}

Submitted 16 May 2012

First decision 10 June 2012

Resubmitted 16 September 2012

Accepted 17 September 2012

\section{SUMMARY}

\section{Background}

There is clear benefit from combination therapy with infliximab and immunosuppressive drugs (IS), but few data are available for adalimumab (ADA).

\section{Aim}

Our aim was to assess the efficacy of ADA monotherapy and ADA+IS for induction and maintenance therapy in Crohn's disease.

\section{Methods}

Retrospective study of patients with Crohn's disease treated with ADA in Oxford, UK or Liège, Belgium. Treatment periods were divided into 6month semesters. A combination therapy semester was defined as ADA+IS for at least 3 months; successful induction meant clinical response; a semester with flare as ADA dose escalation, starting steroids, perianal complication, or surgery; and ADA failure as ADA withdrawal for secondary loss of response or intolerance. Semesters with and without flares were compared through univariate and multivariate analysis.

\section{Results}

Successful induction was achieved in 171/207 (83\%) patients, with no significant difference between $\mathrm{ADA}+\mathrm{IS}$ and $\mathrm{ADA}$ monotherapy ( $85 \%$ vs. $82 \%$, $P=0.50)$. Five hundred and sixty-two semesters in 181 patients were included for maintenance analysis. ADA+IS was not associated with fewer semesters with flare $(34 \%$ vs. $35 \%, P=0.96)$, or with ADA failure $(6 \%$ vs. $8 \%, P=0.43)$. Nevertheless, combination therapy in the first semester was associated with a lower risk of $\mathrm{ADA}$ failure $(5 \%$ vs. $10 \%, P=0.04$, $\mathrm{OR}=0.48)$ and combination therapy beyond 6 months was associated with fewer semesters with flares $(14 \%$ vs. $36 \%, P=0.02$, OR $=0.31)$.

\section{Conclusions}

There may be a benefit from ADA+IS combination therapy during the first semester of initiating ADA, with a slight decrease in ADA failure and lower need for ADA dosage escalation. 


\section{Reenaers et al.}

\section{INTRODUCTION}

The benefit of concommittant oral immunossupressive drugs with sheduled anti-TNF maintenance therapy has been established for infliximab (IFX), ${ }^{1}$ but remains unclear for adalimumab (ADA), although combination therapy is routinely used with all anti-TNF agents in rheumatological practice. ${ }^{2}$ Concommitant immunosuppressive (IS) drugs, including azathioprine (AZA), mercaptopurine (MP), or methotrexate (MTX), appear to reduce the risk of antibody formation to the drugs, which are associated with lower trough levels, shorter time to relapse and infusion reactions. ${ }^{3,4}$ In IS-naive patients with relatively early Crohn's disease treated with IFX, the prospective Study Of biologic and immunomodulator Naive patients In Crohn's disease (SONIC) trial clearly demonstrated the benefit of combination therapy with IFX and AZA. ${ }^{1}$ In patients with longer duration of Crohn's disease, many of whom had already been exposed to IS in clinical practice, a retrospective study from Paris also suggested a potential benefit of IFX+IS combination therapy, at what ever stage IS was combined with IFX. ${ }^{5}$ Few data are available for ADA. In the pivotal registration study of ADA (Crohn's trial of the fully Human Antibody adalimumab for Remission Maintenance, CHARM), post-hoc analysis did not detect any impact of IS co-treatment on the remission rate achieved at 1 year. ${ }^{6}$ The Leuven group also reported no more treatment failure in patients on ADA monotherapy than combination therapy, but a slightly shorter time to drug escalation. ${ }^{7}$ The aim of our study was to assess the impact of $\mathrm{ADA}+\mathrm{IS}$ combination therapy on the rate of response to ADA induction, as well as its effect on flares of Crohn's disease or treatment failure during maintenance therapy with $\mathrm{ADA}$ in routine clinical practice.

\section{METHODS}

\section{Patients}

All patients ever treated with ADA for Crohn's disease at the John Radcliffe Hospital, Oxford, UK and CHU Sart Tilman, Liège, Belgium, were considered for inclusion. The diagnosis of Crohn's disease was based on standard criteria, ${ }^{8}$ with disease distribution and behavior as most recently assessed at the time of ADA therapy. ${ }^{9}$

\section{Study design}

Retrospective analysis of 6-month treatment periods (semesters) for induction success and efficacy of maintenance therapy during a minimum 12-month period, for flare or treatment failure, comparing semesters with and without combination therapy.

\section{Induction success}

For the assessment of response to induction, only patients with at least 3 months' exposure to ADA were considered (Figure 1). Combination therapy for induction was defined as a patient who had received 3 months of $\mathrm{ADA}+\mathrm{IS}$ during the induction period, with IS started at least 3 months before commencing ADA. Induction success was evaluated at 3 months, defined as a clinical response or remission as determined by the gastroenterologist at a routine clinical visit.

\section{Maintenance efficacy}

For the assessment of maintenance efficacy, only patients with at least 12 months' continuous treatment with ADA were analysed (Figure 1). The treatment period was divided into semesters (below). Combination therapy (ADA+IS) for maintenance was defined as a minimum 3 months' IS treatment during a semester, with AZA (2$2.5 \mathrm{mg} / \mathrm{kg}$ ), MP (1-1.5 mg/kg), or MTX (15-25 mg/week administrated orally or by injection). Thiopurine therapy was optimised by weight or leucocyte count, but not by metabolite monitoring.

\section{Semesters}

A semester was defined as a 6-month period with ADA. A flare semester was defined as deterioration in clinical symptoms requiring treatment modification (ADA reinduction, escalation to weekly ADA injection, initiation of corticosteroids, or switch to another biologic), new perianal complication, or abdominal surgery for active CD. A remission semester was a semester without a flare on ADA every other week, or de-escalation from ADA

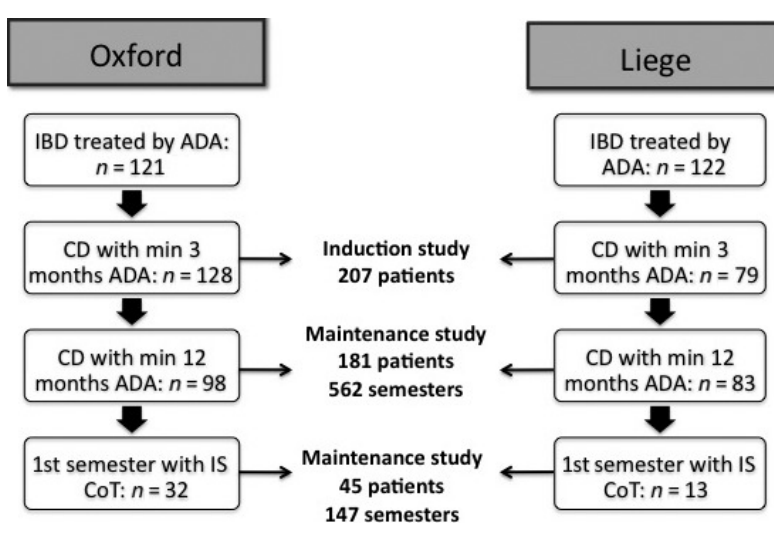

Figure 1 | Patient recruitment. 
weekly to every other week and without a flare during the 2 following months. A failure semester was defined as ADA withdrawal for secondary loss of response, or intolerance. The semester total was the cumulative number of semesters on treatment. ADA interruption was defined as interrupted therapy for $>4$ weeks for any reason (flare, surgery, infection, pregnancy, or cessation during remission). Re-starting ADA was considered to be a new treatment period, so more than one treatment period with $\mathrm{ADA}$ was possible for each patient.

\section{Analysis of semesters}

Analysis of flare semesters and failure semesters compared semesters on ADA monotherapy and ADA+IS combination therapy. Each semester was analysed separately. Overall analysis excluded the first semester and incomplete semesters, with a separate analysis of the subgroup of patients exposed to IS during the first semester of ADA therapy, similar to that performed in a previous study for IFX. ${ }^{5}$ In the group of patients treated with ADA + IS during the first semester, subpopulations of IS failure (who had failed IS treatment in the months before starting $\mathrm{ADA}$ ) and of IS others (meaning those who were naive to IS, or who started IS at the same time as ADA, or who for some reason had ADA started without evidence of active disease, such as post-operatively) could be identified, so were evaluated separately, as well as a comparison between thiopurines (AZA or MP) and MTX.

\section{Statistical analysis}

Descriptive statistics are expressed as mean (standard deviation) for continuous variables and as numbers (percentages) for qualitative variables. Induction success was assessed by comparing proportions achieving clinical response or remission on ADA monotherapy and ADA + IS during induction using Chi-square test. Multivariate logistic regression analysis was performed after univariate analysis had identified factors potentially associated with successful induction.

Maintenance efficacy was assessed by comparing proportions of semesters with flare and failure, for semesters on ADA monotherapy and ADA+IS in the whole population using Chi-squared test.

The risk of flare according to the semester total and type of IS was studied using generalised estimated equations. ${ }^{10}$ Demographic and clinical factors potentially associated with a flare semester or failure semester were first analysed using univariate analysis: the factors were age, gender, weight, age at diagnosis, disease duration, age at diagnosis according to Montréal classification, current
$\mathrm{CD}$ location, current $\mathrm{CD}$ behaviour, current perineal disease, previous surgery, number of previous operations, smoking habit, family history of IBD, spondylarthropathy, previous use of IFX, ADA, or certolizumab pegol, ADA induction regimen, $\mathrm{CRP}$ at $\mathrm{ADA}$ start, $\mathrm{ADA}+\mathrm{IS}$ and $\mathrm{ADA}$ + IS during the first semester, and centre (Oxford/Liège). Variables with a $P$-value $<0.10$ in univariate analysis were considered for multivariate logistic regression analysis. A $P$-value $<0.05$ was considered significant.

The group of patients treated with IS during the first semester of ADA therapy was then analysed using the same approach. Proportions of subsequent flare semesters on ADA monotherapy and ADA+IS semesters and factors associated with flare and failure semesters were studied using univariate and multivariate analysis, using the same factors as the whole population apart from $\mathrm{ADA}+\mathrm{IS}$ during the first semester.

The proportion of semesters with flare on ADA+IS was compared between patients with IS failure and those with IS tolerant at $\mathrm{ADA}$ start using a Chi-squared test. $\mathrm{ADA}+$ thiopurine semesters and $\mathrm{ADA}+\mathrm{MTX}$ semesters were also performed using Chi-squared test. Calculations were performed using the 9.2 version SAS logiciel (SAS Institute, Cary, NC, USA).

\section{RESULTS}

\section{Patient characteristics}

A total of 243 patients with IBD (239 Crohn's disease, 2 ulcerative colitis, 2 IBD unclassified) exposed to ADA were screened for inclusion. Those without a definite diagnosis of Crohn's disease, or who did not meet the criteria of 3 months' treatment with ADA for assessing induction success or 12 months' for maintenance efficacy were excluded. We identified 207 patients suitable for the evaluating induction success (Oxford $n=128$, Liege $n=79$ ) and 181 patients (Oxford $n=98$, Liege $n=83$ ) suitable for evaluating maintenance efficacy (Figure 1). Among these, 45 had received ADA+IS during the first semester and were further analysed separately. Our retrospective analysis identified only one severe adverse event: an episode of severe pneumonia requiring intensive care for 4 days in a patient on ADA monotherapy during the third semester of ADA treatment. Patient characteristics are described in Table 1.

\section{Impact of $A D A+I S$ on the response to ADA induction} $(n=207)$

The rate of successful ADA induction was 171/207 (83\%) at 3 months. ADA was started with IS in 74 patients 


\section{Reenaers et al.}

Table 1| XXXXXXXXXXXXXXXXXXXXXXXXXX

\begin{tabular}{|c|c|c|c|}
\hline Patients characteristics & $\begin{array}{l}\text { Induction study } \\
\qquad N=207 \text { (\%) }\end{array}$ & $\begin{array}{l}\text { Maintenance study: } \\
\text { Patients treated } 12 \text { months } \\
\text { with ADA } \\
N=181(\%)\end{array}$ & $\begin{array}{l}\text { Maintenance study: } \\
\text { Patients treated with ADA+IS } \\
\text { during the 1st semester } \\
N=45(\%)\end{array}$ \\
\hline Age (years, mean \pm s.d.) & $33 \pm 11$ & $33 \pm 11$ & $33 \pm 13$ \\
\hline Male gender & $79(38)$ & $75(41)$ & $19(42)$ \\
\hline Weight (kg) & $67 \pm 16$ & $67 \pm 14$ & $68 \pm 16$ \\
\hline Age at diagnosis (years, mean \pm s.d.) & $22 \pm 9$ & $27 \pm 8$ & $21 \pm 9$ \\
\hline Disease duration (years, mean \pm s.d.) & $11 \pm 8$ & $12 \pm 10$ & $11 \pm 8$ \\
\hline \multicolumn{4}{|l|}{ Age at diagnosis } \\
\hline A1 & $44(21)$ & $44(24)$ & $14(31)$ \\
\hline A2 & $154(74)$ & $131(73)$ & $29(65)$ \\
\hline A3 & $9(5)$ & $6(3)$ & $2(4)$ \\
\hline \multicolumn{4}{|l|}{ Current disease location } \\
\hline L1 & $24(12)$ & $23(12)$ & $2(4)$ \\
\hline L2 & $51(24)$ & $41(23)$ & $12(27)$ \\
\hline L3 & $132(64)$ & $100(55)$ & $24(53)$ \\
\hline$L 4^{\star}$ & $23(11)$ & $19(10)$ & $7(15)$ \\
\hline \multicolumn{4}{|l|}{ Current disease behavior } \\
\hline B1 & $122(59)$ & $94(52)$ & $27(60)$ \\
\hline B2 & $56(27)$ & $55(30)$ & $11(24)$ \\
\hline B3 & $28(14)$ & $32(18)$ & $7(16)$ \\
\hline Active perianal disease & $113(55)$ & $91(50)$ & $26(58)$ \\
\hline Surgery & $68(34)$ & $90(50)$ & $15(33)$ \\
\hline \multicolumn{4}{|l|}{ Number of surgeries } \\
\hline 0 & 102 (49) & $91(50)$ & $30(67)$ \\
\hline 1 & $64(31)$ & $51(28)$ & $6(13)$ \\
\hline 2 & $16(8)$ & $16(9)$ & $3(7)$ \\
\hline$\geq 3$ & $25(12)$ & $23(13)$ & $6(13)$ \\
\hline Smoking habit & $68(34)$ & $66(38)$ & $15(36)$ \\
\hline \multicolumn{4}{|l|}{ Family history of IBD } \\
\hline No & $142(83)$ & $123(81)$ & $28(78)$ \\
\hline 1st degree & $13(8)$ & $11(7)$ & $2(5)$ \\
\hline$\geq 2$ nd degree & $15(9)$ & $18(12)$ & $6(17)$ \\
\hline Spondylarthropathy & $3(1)$ & $5(3)$ & $0(0)$ \\
\hline Previous Infliximab & $132(64)$ & $114(63)$ & $33(73)$ \\
\hline Previous Adalimumab & $25(12)$ & $18(10)$ & $3(7)$ \\
\hline Previous Certolizumab & $14(7)$ & $14(8)$ & $3(7)$ \\
\hline \multicolumn{4}{|l|}{ ADA induction regimen } \\
\hline $160 / 80$ & $142(69)$ & $124(70)$ & $31(72)$ \\
\hline $80 / 40$ & $20(10)$ & $20(11)$ & $6(14)$ \\
\hline none & $33(16)$ & $25(14)$ & $4(9)$ \\
\hline Unknown & $12(5)$ & $12(5)$ & $4(5)$ \\
\hline CRP at ADA start $(\mathrm{mg} / \mathrm{L}$, mean $\pm \mathrm{s} . \mathrm{d})$. & $22 \pm 31$ & $19 \pm 27$ & $15 \pm 25$ \\
\hline ADA+IS during 1st semester & $N A^{\star}$ & $63(35)$ & $45(100)$ \\
\hline ADA+IS semesters & NA & $90(16)$ & $56(38)$ \\
\hline ADA treatment duration (months, mean \pm s.d.) & $44 \pm 51$ & $26 \pm 11$ & $27 \pm 11$ \\
\hline Severe adverse events & $0(0)$ & $1(0.5)$ & $1(2)$ \\
\hline Semesters & NA & 562 & 147 \\
\hline Centre Oxford/Liège & 79/128 (38/62) & $98 / 83(54 / 46)$ & $32 / 13(71 / 29)$ \\
\hline
\end{tabular}

No patients had isolated L4; all patients with L4 had concomittent L1, L2 or L3 involvement.

* NA, Non applicable.

(36\%) and the response to ADA induction did not differ between the group started on ADA monotherapy and the group started on $\mathrm{ADA}+\mathrm{IS}$ ( $82 \%$ and $85 \%$ respectively, $p=0.50)$. This group included those $(n=47)$ already on IS for $\geq 3$ months at commencement of $\mathrm{ADA}$, subject to separate analysis. In the multivariate analysis, two inde- 
pendent factors were associated with failure of $\mathrm{ADA}$ induction: high CRP $(44 \mathrm{mg} / \mathrm{L}$ vs. $18 \mathrm{mg} / \mathrm{L}, P=0.01$, $\mathrm{OR}=1.02,95 \%$ CI: $1.01-1.03)$ and age under 16 at diagnosis $(P=0.02$, OR $=4.08,95 \%$ CI: $1.49-11.15)$.

Impact of ADA+IS on maintenance in the whole population $(n=181)$

Five hundred and sixty-two semesters were analyzed, including 472 semesters on ADA monotherapy and 90 semesters on ADA+IS (thiopurine $n=65$, MTX $n=25$ ). The mean semester total for follow-up was 4 (range: 29).

The rate of semesters with flare in the whole population was $35 \%(n=195)$ and no difference was observed between semesters with ADA monotherapy and semesters with ADA+IS (35\% and $34 \%$ respectively, $P=0.96$ ) (Figure 2a). The large majority of semesters with flare corresponded to the need for escalation to ADA weekly dosing $(n=157,81 \%)$. We identified 18 semesters with surgery (9\%), 19 with new perianal complications (10\%), 1 with a switch to IFX $(0.01 \%), 12$ with ADA reinduction (7\%) and 20 with corticosteroid prescriptions (10\%). No significant differences were observed regarding the need for surgery $(P=0.05)$ or new perianal complications $(P=0.21)$ between ADA monotherapy or ADA+IS (Table 2). There were no more failures in ADA+IS semesters compared with ADA monotherapy semesters ( $6 \%$ and $8 \%$ respectively, $P=0.43$ ) in the whole population (Figure 3a). The rate of flare semesters was stable over time $(P=0.90)$ and did not change according to $\mathrm{ADA} \pm \mathrm{IS}(P=0.08)$. In multivariate analysis, semesters with flares were associated with four independent factors: active perianal disease $(P=0.02, \mathrm{OR}=1.57,95 \% \mathrm{CI}$ : $1.07-2.32)$, previous surgery $(P<0.01, \mathrm{OR}=1.89,95 \%$ CI: $1.31-2.87)$, female gender $(P=0.01, \mathrm{OR}=1.68,95 \%$ CI: $1.12-2.52)$ and Liège centre $(P<0.01, \mathrm{OR}=1.94$, 95\% CI: 1.31-2.87). Independent factors associated with the risk of new perianal complications and abdominal surgery are shown in Table 3. ADA+IS was not an independent parameter associated with flare semester. Concerning $\mathrm{ADA}$ failure, the only independent factor after multivariate analysis was ADA+IS during the first semester $(P=0.04$, OR $=0.48,95 \%$ CI: $0.24-0.97)$. No

Table 2 | All CD patients $(n=181)$ : Semesters with IBD activity according to $A D A \pm I S$. The rate of semesters with flare was not significantly different between patients treated with ADA monotherapy and patients treated with ADA+IS. Regarding the type of flare, surgery, new perianal complication or ADA dose escalation, no difference was demonstrated between the 2 groups

\begin{tabular}{llll}
\hline$N(\%)$ & ADA monotherapy & ADA+IS & $P$-value \\
\hline IBD flare & $164(35)$ & $31(34)$ & 0.96 \\
Surgery & $12(7)$ & $6(19)$ & 0.06 \\
Perianal complications & $18(11)$ & $1(3)$ & 0.2 \\
ADA dose escalation & $104(81)$ & $53(80)$ & 0.95 \\
\hline
\end{tabular}

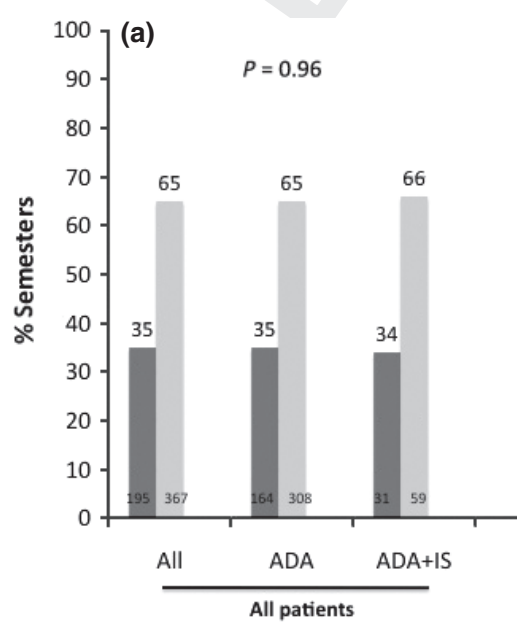

(b)
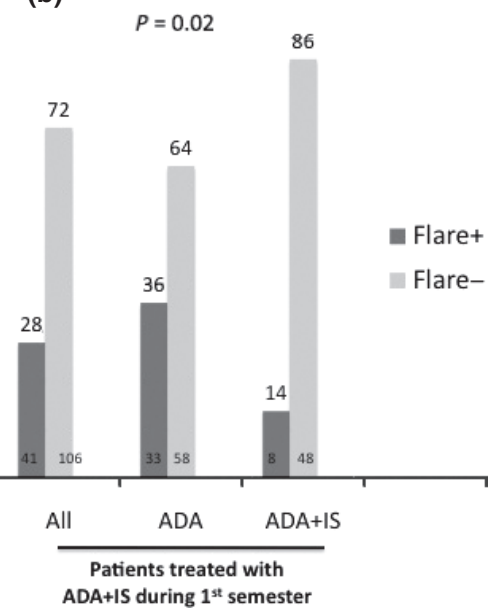

Figure 2 | Semesters with flare according to ADA \pm IS. (a). In the whole population ( 562 semesters in 181 patients), the rate of semesters with flare was not statistically different between semesters with ADA monotherapy and ADA+IS $(P=0.96)$. (b) In the patients treated with ADA+IS during the first semester (147 semesters in 45 patients), flares were statistically less frequent in semesters with ADA+IS (14\%) compared to ADA monotherapy $(36 \%)(P=0.02)$. 


\section{Reenaers et al.}

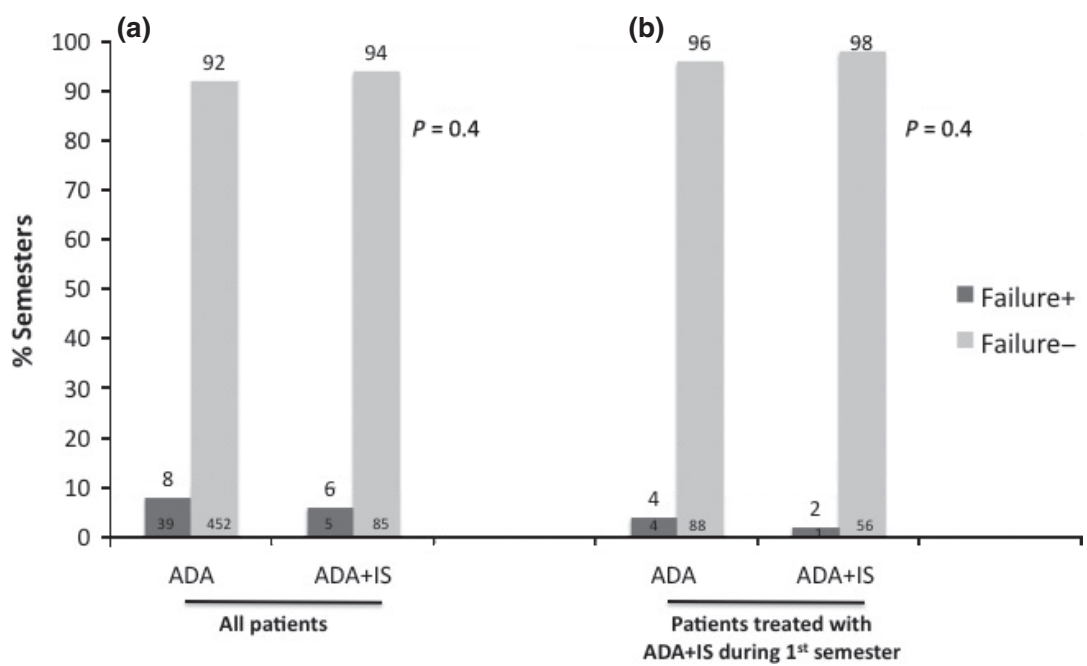

Figure 3 | Semesters with ADA failure according to ADA \pm IS. In the whole population (a) (562 semesters in 181 patients) and in the patients treated with ADA+IS during the first semester (b) (147 semesters in 45 patients), the rate of semesters with ADA failure was not statistically different between semesters with ADA monotherapy and ADA+IS $(P=0.4)$.

Table 3 | Independant risk factors for surgery and perianal complication in the whole population $(n=181)$

OR (95\% Confidence $P$-value limits)

\begin{tabular}{lrc}
\hline Surgery $(n=18)$ & & \\
Male gender & $<0.01$ & $13.16(3.29-52.63)$ \\
Disease duration & 0.01 & $1.12(1.01-1.21)$ \\
Active perianal disease & 0.02 & $5.51(1.30-23.15)$ \\
Oxford centre & $<0.01$ & $8.8(2.29-34.24)$ \\
Perianal flare $(n=19)$ & & \\
Active perianal disease & 0.01 & $7.74(1.65-36.22)$ \\
Oxford centre & 0.03 & $3.33(1.14-9.77)$ \\
\hline
\end{tabular}

other independent factor, including semester with ADA + IS, was identified. The risk of semester with failure was $10 \%$ vs. $5 \%$ in patients with $\mathrm{ADA}$ monotherapy in the first semester and ADA+IS respectively.

Impact of ADA+IS on maintenance in patients treated during the first semester with ADA+IS $(n=45)$

Forty-five patients received ADA+IS during the first semester and were analysed separately (147 semesters, including 91 subsequent semesters with ADA monotherapy and 56 with $\mathrm{ADA}+\mathrm{IS}$ ). Analysis of this subgroup of patients demonstrated that flares were less frequent in semesters with continued ADA+IS compared with semesters with ADA monotherapy (14\% and $36 \%$ respectively, $P=0.02, \quad$ OR $=0.31,95 \%$ CI: $0.12-0.81$ )
(Figure 2b). The rate of flare did not change over time $(P=0.86)$ and the protective effect of ADA+IS was stable according to the semester total $(P<0.01)$ (Figure 4). Flare semester characteristics were as followed: ADA weekly $(n=35 / 41,86 \%)$, abdominal surgery $(n=3$, $7 \%)$, perianal complications $(n=3,7 \%)$, corticosteroids $(n=22 \%)$. There were no more semesters with ADA failures in semesters with ADA+IS compared with semesters with ADA monotherapy ( $2 \%$ and $4 \%$ respectively, $P=0.41$ ) (Figure $3 \mathrm{~b}$ ). Among those patients on IS at initiation of $\mathrm{ADA}$, the proportion of semesters with flare did not differ between patient having failed IS or not before ADA start $(P=0.86)$. Futhermore, there was no significant difference between semesters with ADA + thiopurine and $\mathrm{ADA}+\mathrm{MTX}(P=0.84)$.

In multivariate analysis, three independent factors were significantly associated with flare semesters: ADA monotherapy $(P=0.02, \mathrm{OR}=3.28,95 \% \mathrm{CI}: 1.25-8.3)$, previous surgery $(P=0.01, \mathrm{OR}=3.69,95 \% \mathrm{CI}: 157-$ $8.68)$ and Liège centre $(P=0.01, \mathrm{OR}=3.17,95 \% \mathrm{CI}$ : $1.40-7.19)$. Factors specifically associated with the risk of new perianal complications and abdominal surgery were not analysed because of the small number of events in this subgroup. No clinical or demographic factors were associated with ADA failure.

\section{DISCUSSION}

Although the benefit of IS in patients treated with IFX has been clearly demonstrated both in IS naive and IS 


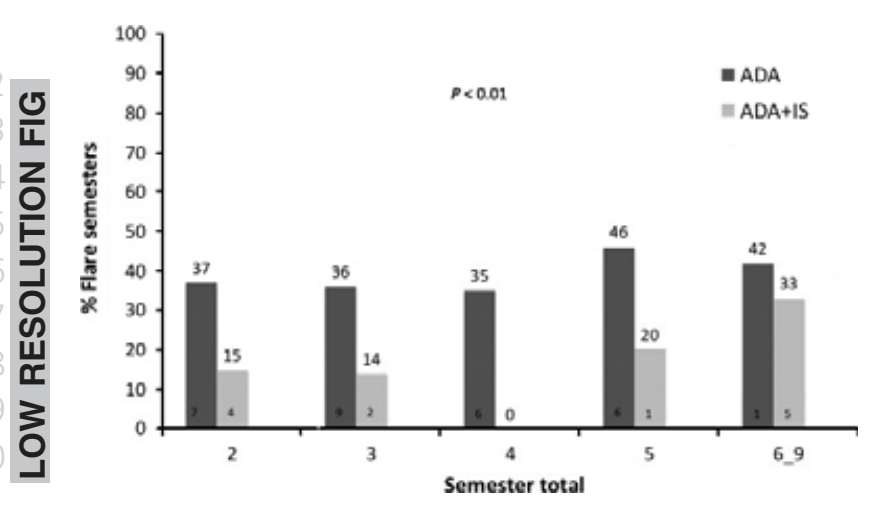

Figure 4 | Semesters with flare according to $A D A \pm I S$ and semesters total in the patients treated with ADA +IS during the first semester (147 semesters in 45 patients). The rate of semesters with flare was stable over the time $(P=0.86)$. The protective effect of ADA +IS compared to ADA monotherapy for a flare semester did not change according to the semester total $(P<0.01)$.

exposed patients, the benefit of such combination therapy is not well documented with ADA. In this retrospective analysis, we could not show any benefit of ADA+IS for achieving induction success for clinical response and remission. In maintenance ADA therapy, we could also not show any decrease in semesters with flares during semesters with ADA+IS combination therapy. This is consistent with the results of the subanalysis of the CHARM trial, ${ }^{6}$ but at odds with the practice-based French study on IFX. ${ }^{5}$

Nevertheless, there was a benefit of combination therapy (ADA+IS) when IS had been given during the first semester of ADA treatment. This was associated with half the rate of ADA failure over the subsequent semesters. Although this was quantatively small (from $10 \%$ to $5 \%$ ), it is likely to be clinically as well as statistically significant. Furthermore, among these patients treated during the first semester with $\mathrm{ADA}+\mathrm{IS}$, there were fewer semesters with flares during subsequent maintenance treatment with $\mathrm{ADA}+\mathrm{IS}$ compared with semesters on ADA monotherapy, suggesting a sustained benefit.

The large majority of semester flares were characterised by escalation in ADA dosage. On subanalysis, it was only prevention of dose escalation with ADA that benefited from continued ADA+IS combination therapy after having been treated by this combination in the first semester, not other definitions of flares, including new perianal complications or abdominal surgery, although numbers (19 and 18/195 semesters with abdominal oper- ations and new perianal complications respectively) in the latter groups were very small. A similar benefit of $\mathrm{ADA}+\mathrm{IS}$ was suspected by the Leuven group who noted a shorter time to ADA dose escalation, but no excess of treatment failure on ADA monotherapy. ${ }^{7}$

Combination therapy with IS can reduce anti-TNF antibody formation and improve the pharmacokinetics of anti-TNF drugs. ${ }^{11,12}$ It has also been demonstrated that discontinuation of $\mathrm{ADA}$ due to treatment failure is associated with low ADA trough level concentrations. ${ }^{7}$ In our study, the benefit of combination therapy at the time of starting ADA, whether or not continued after the first semester, might be explained by early inhibition of anti-ADA antibody formation, leading to higher trough levels from the start of the therapy that could predict long-term efficacy of ADA. An alternative explanation might simply be better control of the inflammation on combination therapy from the onset of treatment that reduces the risk of relapse compared with ADA monotherapy. Prospective studies combined with anti-ADA antibody and ADA trough level measurements are required to validate these hypotheses.

Apart from combination therapy in the first semester, among the factors associated with flare semesters, were previous surgery and being treated in Liège. Although previous abdominal surgery is consistent with more severe disease, ${ }^{11}$ the association with Liège deserves a specific comment. In the current study, fewer in the Belgian cohort received IS than the British cohort, but weekly treatment with ADA was more common in Belgium. As the commonest reason for defining a flare was dose escalation on monotherapy, this treatment strategy is reflected in the increased rate of flares in Liège compared to Oxford. In contrast, surgery and perianal complications were more frequent in Oxford, but accounted for only a small proportion of flares. These results may reflect easier access to anti-TNF therapy in Belgium. Indeed, the Belgian healthcare system allows easier access to long-term anti-TNF therapy as well as dose escalation, whereas the British healthcare system encourages shorter treatment duration with a higher threshold for dose escalation. This might explain why, in similar clinical circumstances, patients may be treated in Belgium with weekly ADA monotherapy, but in the UK with ADA every other week in combination with IS or even surgery.

Our study was closely modelled on that of Sokol et al., ${ }^{5}$ who demonstrated a benefit of IS+IFX compared with IFX monotherapy using semester-based analysis. However, in their study, only patients receiving IFX+IS 


\section{Reenaers et al.}

during the first semester were included, which corresponds to our subgroup of 45 patients (157 semesters) treated with $\mathrm{ADA}+\mathrm{IS}$ during the first semester. In their study, IFX+IS was associated with fewer semesters with dose escalation or interval reduction compared with subsequent semesters on IFX monotherapy. In this respect, we confirm a similar result in those on ADA+IS during the first semester, with fewer needing ADA dose escalation in subsequent ADA+IS semesters compared with ADA monotherapy. The Sokol study ${ }^{5}$ also reported fewer perianal complications or switches in biological therapy in IFX+IS semesters, but no impact on the frequency of semesters with abdominal surgery. These events were too rare to analyse in our population. Furthermore, $70 \%$ of our patients had already been treated by another antiTNF agent (usually IFX) before ADA; hence, switch biological therapy in the Sokol study might be compared with ADA failure in our study. Although Sokol et al. ${ }^{5}$ found a modest decrease in the need to switch to another biologic during IFX+IS semesters, we found no benefit of prolonged ADA+IS beyond the first semester. This is more comparable to the results in the Leuven cohort and IMID trial. ${ }^{12}$

Our study has several limitations due mainly to its retrospective nature. First, the definition of the flare was retrospective and included different clinical situations (e. g. starting steroids, dose escalation, surgery, etc.). Second, patients were not randomised between ADA monotherapy and ADA+IS, hence these two populations are not strictly comparable. Third, there were no serum samples to measure trough levels or anti-ADA antibodies. Fourth, data on the intake and the dose of corticosteroid during the induction time with ADA were missing for half of the patients, although just $5 \%$ of all data in the induction group were missing. Although the available data did not show any influence of corticosteroids on the response to ADA induction, an effect cannot be excluded; hence, a lack of influence of steroids on response to ADA cannot be concluded. Nevertheless, in this retrospective analysis from the experience of two referral centers, $q$ the benefit of ADA+IS appears limited to those co-treated at the start of ADA therapy. Combination therapy with ADA during the first semester slightly decreased the rate of ADA failure during maintenance therapy independently of whether ADA+IS was continued beyond the first semester. During maintenance therapy, ADA+IS was associated with a lower rate of ADA dose escalation.

We think our results may help clinicians decide whether to use ADA in monotherapy or combination therapy, particularly, with regard to the potential benefits from such combination at the start of ADA. Accordingly, it seems reasonable to start with the combination and then continue ADA monotherapy after about 6 months of combination therapy, as the need for dose escalation does not appear to be affected after the first semester. The choice of the treatment strategy needs to be balanced with potential risks of infection and cancer and also with the cost of the strategy. In older patients with high comorbidity, or younger male subjects at increased risk of hepatosplenic T-cell lymphoma ${ }^{14}$ on combination therapy, stopping the IS 6 months after ADA start seems a reasonable option. It is also not unreasonable to give $\mathrm{ADA}$ as monotherapy from the start as long as the slight increase in the absolute risk of treatment failure is recognised. A randomised trial is needed to confirm whether ADA+IS is more effective than ADA monotherapy, particularly to examine whether any benefit is limited to combination therapy in the first semester, and whether it simply decreases the need for ADA dose escalation, or whether it also decreases clinically relevant measures of patient outcome.

\section{ACKNOWLEDGEMENT}

Declaration of personal and funding interests: None.

\section{REFERENCES}

1. Colombel JF, Sandborn WJ, Reinisch $\mathrm{W}$, et al. Infliximab, azathioprine, or combination therapy for Crohn's disease. N Engl J Med 2010; 362: 1383 95.

2. Nam JL, Winthrop KL, van Vollenhoven RF, et al. Current evidence for the management of rheumatoid arthritis with biological diseasemodifying antirheumatic drugs: a systematic literature review informing the EULAR recommendations for the management of RA. Ann Rheum Dis 2010; 69: 976-86.

3. Vermeire S, Noman M, Van Assche G, et al. Autoimmunity associated with anti-tumor necrosis factor alpha treatment in Crohn's disease: a prospective cohort study. Gastroenterology 2003; 125: 32-9.

4. Baert F, Noman M, Vermeire S, et al. Influence of immunogenicity on the long-term efficacy of infliximab in Crohn's disease. N Engl J Med 2003; 348: 601-8.

5. Sokol H, Seksik P, Carrat F, et al. Usefulness of co-treatment with immunomodulators in patients with inflammatory bowel disease treated with scheduled infliximab maintenance therapy. Gut 2010; 59: 1363-8.

6. Colombel JF, Sandborn WJ, Panaccione $\mathrm{R}$, et al. Comparison of two 


\section{Co-treatment with immunosuppressors and adalimumab in Crohn's disease}

adalimumab treatment schedule strategies for moderate-to-severe Crohn's disease: results from the CHARM trial. Inflamm Bowel Dis 2009; 15: 1308-19.

7. Karmiris K, Paintaud G, Noman M, et al. Influence of trough serum levels and immunogenicity on long-term outcome of adalimumab therapy in Crohn's disease. Gastroenterology 2009; 137: $1628-40$.

8. Van Assche G, Dignass A, Panes J; For the European Crohn's Colitis Organisation (ECCO), et al. The second European evidence-based Consensus on the diagnosis and management of Crohn's disease: definitions and diagnosis. J Crohns Colitis 2010; 4: 727.
9. Silverberg MS, Satsangi J, Ahmad T, et al. Toward an integrated clinical, molecular and serological classification of inflammatory bowel disease: report of a Working Party of the 2005 Montreal World Congress of Gastroenterology. Can J Gastroenterol 2005; 19(Suppl. A): 5-36.

10. Omar RZ, Thompson SG. Analysis of a cluster randomized trial with binary outcome data using a multi-level model. Stat Med 2000; 19: 2675-88.

11. Vermeire S, Noman M, Van Assche G, et al. Effectiveness of concomitant immunosuppressive therapy in suppressing the formation of antibodies to infliximab in Crohn's disease. Gut 2007; 56: 1226-31.
12. Van Assche G, Magdelaine-Beuzelin C, D'Haens G, et al. Withdrawal of immunosuppression in Crohn's disease treated with scheduled infliximab maintenance: a randomized trial. Gastroenterology 2008; 134: 1861-8.

13. Peyrin-Biroulet L, Loftus EV Jr, Colombel JF, Sandborn WJ. The natural history of adult Crohn's disease in population-based cohorts. Am J

2 Gastroenterol 2010; 105: 289-97.

14. Beaugerie L, Brousse N, Bouvier AM, et al. Lymphoproliferative disorders in patients receiving thiopurine for inflammatory bowel disease: a prospective observational study. Lancet 2009; 374: 1617-25. 


\section{Author Query Form}

Journal: $\quad$ APT

Article: $\quad 12076$

Dear Author,

During the copy-editing of your paper, the following queries arose. Please respond to these by marking up your proofs with the necessary changes/additions. Please write your answers on the query sheet if there is insufficient space on the page proofs. Please write clearly and follow the conventions shown on the attached corrections sheet. If returning the proof by fax do not write too close to the paper's edge. Please remember that illegible mark-ups may delay publication. Many thanks for your assistance.

\begin{tabular}{|c|c|c|}
\hline Query reference & Query & Remarks \\
\hline 1 & $\begin{array}{l}\text { AUTHOR: It is AP\&T's policy that all personal and funding interests dur- } \\
\text { ing the last } 2 \text { years are declared. Please consider your interests, and adjust } \\
\text { these statements, or insert similar statements for other interests. } \\
\text { PERSONAL AND FUNDING INTERESTS } \\
\text { IF NONE, state 'None'. } \\
\text { OR, complete relevant parts of these or similar statements: } \\
\text { Authors' declaration of personal interests: } \\
\text { [Name of individual] has served as a speaker, a consultant and an advisory } \\
\text { board member for [names of organisations], and has received research } \\
\text { funding from [names of organisation]. [Name of individual] is an } \\
\text { employee of [Name of organisation]. [Name of individual] owns stocks } \\
\text { and shares in [name of organisation]. [Name of individual] owns patent } \\
\text { [patent identification and brief description]. } \\
\text { Declaration of funding interests: } \\
\text { This study was funded [in part or in full] by [insert name of funding } \\
\text { organisation and provide funding identification numbers], grant number } \\
\text { [insert grant or other identification number]. The [writing or preparation] } \\
\text { of this paper was funded in part by [insert name of funding organisation]. } \\
\text { Initial data analyses were undertaken by [name of individuals if not listed } \\
\text { as authors] who are employees of [name company] and received funding } \\
\text { from [insert name of funding organisation]. Writing support was pro- } \\
\text { vided by [insert name of individual(s)] of [name company] and funded by } \\
\text { [insert name of funding organisation]. }\end{array}$ & \\
\hline 2 & $\begin{array}{l}\text { AUTHOR: Reference [13] has not been cited in the text. Please indicate } \\
\text { where it should be cited; or delete from the Reference List and renumber } \\
\text { the References in the text and Reference List. }\end{array}$ & \\
\hline 3 & AUTHOR: Please provide a suitable legend for Table 1. & \\
\hline 4 & $\begin{array}{l}\text { AUTHOR: Figure } 1 \text { has been saved at a low resolution of } 176 \mathrm{dpi} \text {. Please } \\
\text { resupply at } 600 \mathrm{dpi} \text {. Check required artwork specifications at http:// } \\
\text { authorservices.wiley.com/submit_illust.asp?site=1 }\end{array}$ & \\
\hline 5 & $\begin{array}{l}\text { AUTHOR: Figure } 2 \text { has been saved at a low resolution of } 157 \text { dpi. Please } \\
\text { resupply at } 600 \text { dpi. Check required artwork specifications at http:// } \\
\text { authorservices.wiley.com/submit_illust.asp?site=1 }\end{array}$ & \\
\hline
\end{tabular}




\begin{tabular}{|l|l|l|}
\hline 6 & $\begin{array}{l}\text { AUTHOR: Figure } 3 \mathrm{has} \text { been saved at a low resolution of } 152 \mathrm{dpi} \text {. Please } \\
\text { resupply at } 600 \mathrm{dpi} \text {. Check required artwork specifications at http:// } \\
\text { authorservices.wiley.com/submit_illust.asp?site=1 }\end{array}$ & $\begin{array}{l}\text { AUTHOR: Figure } 4 \mathrm{has} \text { been saved at a low resolution of } 194 \mathrm{dpi} . \text { Please } \\
\text { resupply at } 600 \mathrm{dpi} \text {. Check required artwork specifications at http:// } \\
\text { authorservices.wiley.com/submit_illust.asp?site=1 }\end{array}$ \\
\hline 7 &
\end{tabular}


Required software to e-Annotate PDFs: Adobe Acrobat Professional or Adobe Reader (version 7.0 or above). (Note that this document uses screenshots from Adobe Reader $\mathbf{X}$ )

The latest version of Acrobat Reader can be downloaded for free at: http://get.adobe.com/uk/readerl

Once you have Acrobat Reader open on your computer, click on the Comment tab at the right of the toolbar:

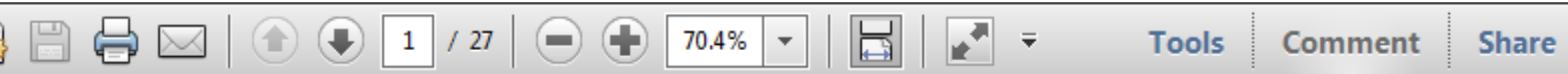

This will open up a panel down the right side of the document. The majority of tools you will use for annotating your proof will be in the Annotations section, pictured opposite. We've picked out some of these tools below:

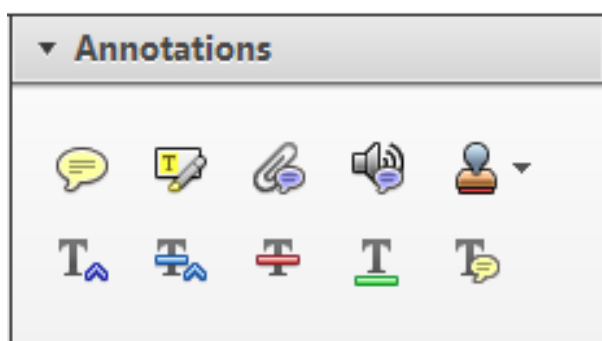

1. Replace (Ins) Tool - for replacing text.

Strikes a line through text and opens up a text box where replacement text can be entered.

How to use it

- Highlight a word or sentence

- Click on the Replace (Ins) icon in the Annotations section.

- Type the replacement text into the blue box that appears.

Idard tramework for the analysis of $\mathrm{m}$ icy-Nevertheless, it also led to exog،

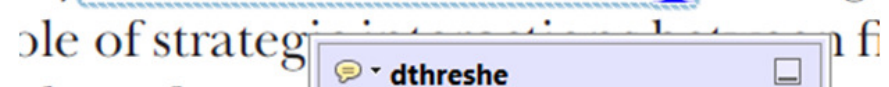
aber of comp 08/06/2011 15:58:17 is that the $\mathrm{s} 1 \overline{\text {, which led }}$ of nain compo: be level, are exc nc

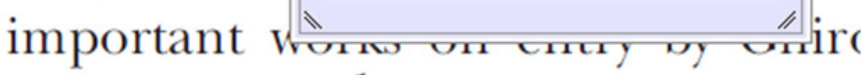
M henceforth) ${ }^{1}$ we snen the "hlark $\mathrm{h}$

3. Add note to text Tool - for highlighting a section to be changed to bold or italic.

Th Highlights text in yellow and opens up a text box where comments can be entered.

\section{How to use it}

- Highlight the relevant section of text.

- Click on the Add note to text icon in the Annotations section.

- Type instruction on what should be changed regarding the text into the yellow box that appears

namic responses of mark ups ent with the VAR evidence

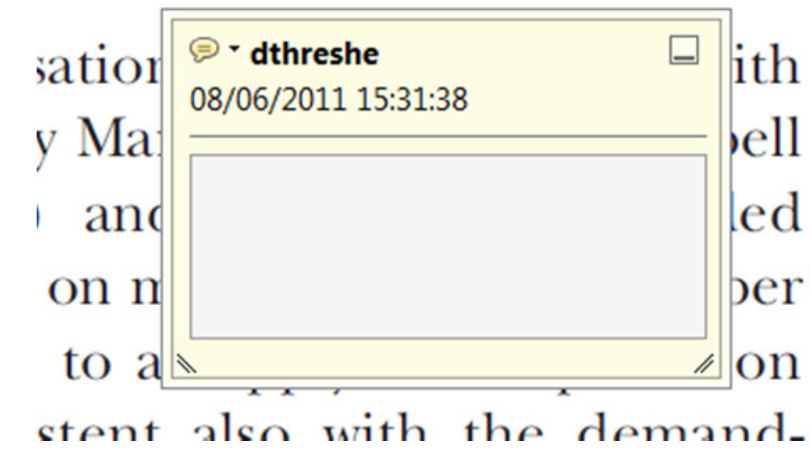

2. Strikethrough (Del) Tool - for deleting text.

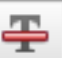

Strikes a red line through text that is to be deleted.

How to use it

- Highlight a word or sentence.

- Click on the Strikethrough (Del) icon in the Annotations section.

there is no room tor extra prohts al s ups are zero and the number of ret) values are not determined by Blanchard and Kiyotaki (1987), sfect competition in general equilil ts of aggregate demand and supply lassical framework assuming mono sen on evorenous nimher of firms

4. Add sticky note Tool - for making notes at specific points in the text.

Marks a point in the proof where a comment needs to be highlighted.

How to use it

- Click on the Add sticky note icon in the Annotations section.

- Click at the point in the proof where the comment should be inserted.

- Type the comment into the yellow box that appears.

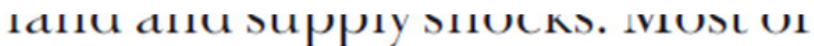

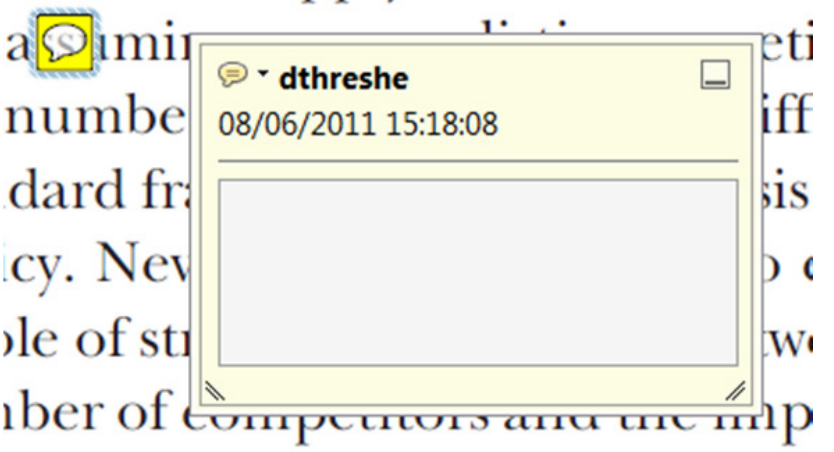

is that the structure of the sects. 
5. Attach File Tool - for inserting large amounts of text or replacement figures.

Inserts an icon linking to the attached file in the appropriate pace in the text.

How to use it

- Click on the Attach File icon in the Annotations section.

- Click on the proof to where you'd like the attached file to be linked.

- Select the file to be attached from your computer or network.

- Select the colour and type of icon that will appear in the proof. Click OK.

E N D

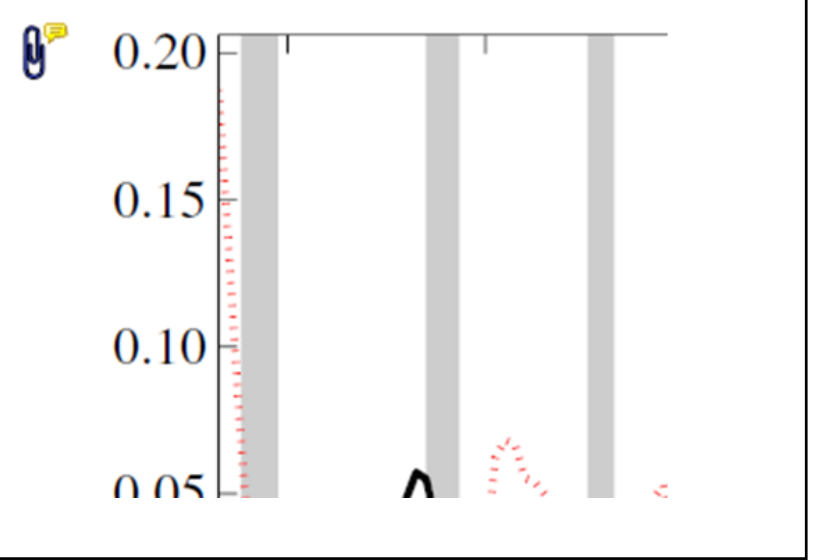

6. Add stamp Tool - for approving a proof if no corrections are required.

- Inserts a selected stamp onto an appropriate place in the proof

\section{How to use it}

- Click on the Add stamp icon in the Annotations section.

- Select the stamp you want to use. (The Approved stamp is usually available directly in the menu that appears).

- Click on the proof where you'd like the stamp to appear. (Where a proof is to be approved as it is, this would normally be on the first page).

of the Dusiness cycie, starting with the on perfect competition, constant ret

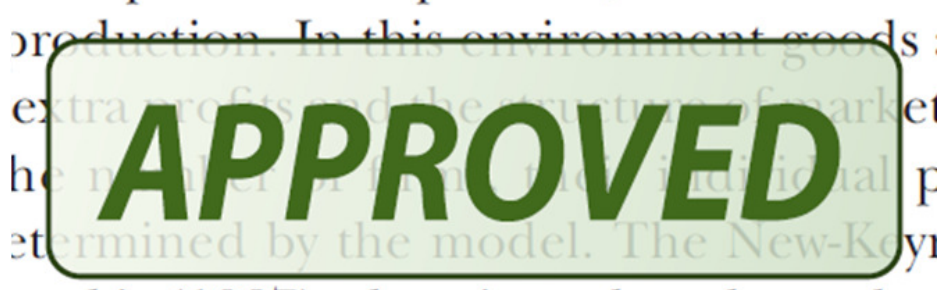
otaki (1987), has introduced produc general equilibrium models with nomin:

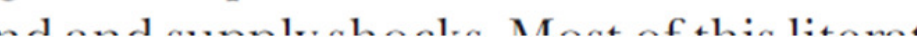

- Drawing Markups

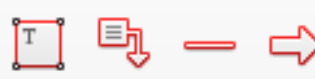

$0 \square \sqrt{ } \rightarrow 0$

\section{How to use it}

- Click on one of the shapes in the Drawing Markups section.

- Click on the proof at the relevant point and draw the selected shape with the cursor.

- To add a comment to the drawn shape, move the cursor over the shape until an arrowhead appears.

- Double click on the shape and type any text in the red box that appears.
7. Drawing Markups Tools - for drawing shapes, lines and freeform annotations on proofs and commenting on these marks.

Allows shapes, lines and freeform annotations to be drawn on proofs and for comment to be made on these marks.

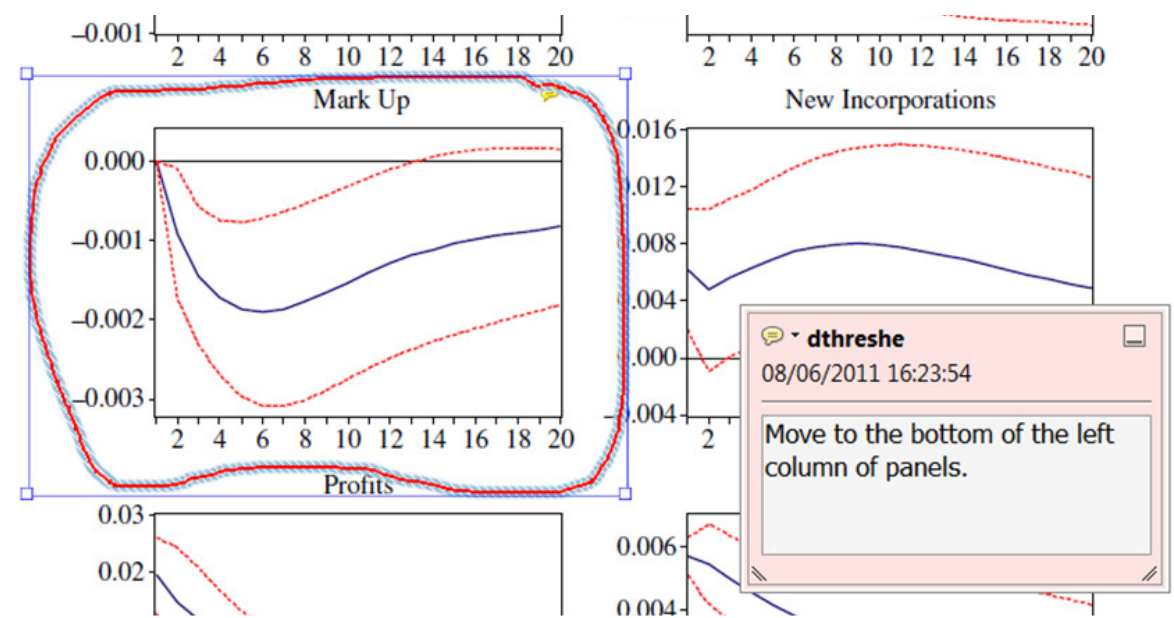

For further information on how to annotate proofs, click on the Help menu to reveal a list of further options:

\begin{tabular}{|l|l|l|l|l|l|l|}
\hline File ecoj_2384_CrxRev2_EV_19-Jul-10.pdf - Adobe Reader & Edit View Window \\
\hline
\end{tabular}

